\title{
An Improved Harmonic Load Flow Formulation
}

Asim Rashid, Juan José Mesas, Luis Sainz

Polytechnical University of Catalonia

Barcelona, Spain

asim.rashid@upc.edu

sainz@ee.upc.edu

Department of Electrical Engineering

Av. Diagonal 647, 08028 Barcelona

juan.jose.mesas@upc.edu

Department of Electrical Engineering

Av. Eduard Maristany 16, 08019 Barcelona 


\title{
An Improved Harmonic Load Flow Formulation
}

\begin{abstract}
The paper contributes an improved harmonic load flow formulation with fewer convergence problems but the same accurate results as traditional formulations. The proposed formulation approaches the harmonic load flow problem as a single nonlinear equation system where the harmonic bus voltage influence on nonlinear load behaviour is considered and harmonic bus voltages at linear buses are not included as unknowns. This formulation allows any sort of nonlinear load to be considered and uses the Newton-Raphson method with true Jacobian matrix to reduce the inherent increase in the number of iterations caused by the presence of highly distorted bus voltages. The numerical results obtained when solving a three-bus network operating under highly distorted bus voltages using traditional harmonic load flow formulations and the improved formulation are comparatively discussed.
\end{abstract}

Keywords: harmonic load flow; power system harmonics; nonlinear equations; harmonic distortion

\section{Nomenclature}

CHLF Complete Harmonic Load Flow

$\mathrm{CHLF}_{\mathrm{m}} \quad$ Complete Harmonic Load Flow (modified)

EMTP Electromagnetic Transients Program

FLF Fundamental Load Flow

$\mathrm{FLF}_{\mathrm{m}} \quad$ Fundamental Load Flow (modified)

HA Harmonic Analysis

HD Harmonic Distortion (individual)

HLF Harmonic Load Flow

HP Harmonic Penetration

HVDC High-Voltage Direct Current

IUHLF Improved Unified Harmonic Load Flow

LED Light-Emitting Diode

NL Nonlinear

NLL Nonlinear Load

SHLF Simplified Harmonic Load Flow

THD Total Harmonic Distortion 
UHLF Unified Harmonic Load Flow

VN Voltage Node method

$i / j / l \quad$ indexes for buses

$h / k \quad$ indexes for harmonic $(h) /$ fundamental and harmonic $(k)$ orders

$m \quad$ index for NLL data

$r \quad$ index for NLL state variables

$\underline{Y}_{i j}^{h} / \underline{Y}_{i j}^{k} \quad$ elements of harmonic $(h) /$ fundamental and harmonic $(k)$ bus admittance matrices

$\underline{V}_{i}^{h} / \underline{V}_{i}^{k} \quad$ harmonic $(h) /$ fundamental and harmonic $(k)$ bus voltages

$\underline{I}_{i}^{h} / \underline{I}_{i}^{k} \quad$ injected harmonic $(h) /$ fundamental and harmonic $(k)$ bus currents

$D^{m}{ }_{i} \quad$ NLL data

$\delta_{i}^{r} \quad \quad$ NLL state variables

$\underline{E}_{T h i}^{h} / \underline{E}_{T h i}^{k} \quad$ harmonic $(h) /$ fundamental and harmonic $(k)$ Thévenin equivalent circuit voltages

$\underline{Z}_{T h i j}^{k} \quad$ elements of fundamental and harmonic $(k)$ Thévenin equivalent circuit matrices

$\mathbf{Y}^{h}{ }_{\mathbf{B}} / \mathbf{Y}^{k}{ }_{\mathbf{B}} \quad$ harmonic $(h) /$ fundamental and harmonic $(k)$ bus admittance matrices $\mathbf{V}_{\mathbf{B}}^{h} \quad$ harmonic bus voltage vectors

$\mathbf{I}_{\mathbf{B}}^{h} \quad$ injected harmonic bus current vectors

$D F, D G \quad$ Jacobian matrices of the nonlinear equation systems $F, G$ 


\section{Introduction}

In the last years, the increasing number of nonlinear loads (NLLs) connected to electric power systems has led to increased harmonic distortion in network voltages and currents. For this reason, the steady state harmonic problem has been extensively studied to determine electric power system harmonic distortion [1]. Procedures for analysing this problem can be divided into time [2] and frequency domain [3-11] procedures. The former, such as Electromagnetic Transients Program (EMTP), are based on the numerical resolution of electric power system differential equations. They treat NLL equations directly, but require a high calculation effort to obtain steady state solutions and difficult management of power consumption loads. The latter, known as harmonic load flow (HLF) formulations, are reformulations of fundamental load flow (FLF) which consider harmonic bus voltages and NLL state variables as additional unknowns to the fundamental bus voltages. Their main drawback is that NLL equations must be adapted to the frequency domain formulation. These frequency domain procedures pose nonlinear equation systems which must be numerically solved to directly obtain the fundamental and harmonic bus voltages of the network and the variables characterising the NLL state. There are also hybrid procedures which work in both domains by using their respective advantages $[12,13]$. The procedures in the frequency domain are the most widely used in the literature, and Newton-Raphson is the most commonly employed numerical method to solve their nonlinear equation systems. Nevertheless, the numerical resolution of these equation systems has several difficulties such as long execution time, convergence problems and large computer memory requirements due to the significant number of involved unknowns [14, 15]. 
Most HLF formulations look for a compromise between simplicity and reliability. The simplest formulations [3-7] assume no harmonic interaction between the electrical network and NLLs, but have some limitations in terms of accuracy if harmonic bus voltages in the network exceed a certain level, or NLLs in the network are very sensitive to harmonic bus voltages. Therefore, other formulations [8-11] which consider the harmonic bus voltage influence on NLL behaviour have been developed. It must be noted that formulations in $[8,10,11]$ do not include the harmonic bus voltages at linear buses as unknowns in the nonlinear equation system, whereas formulation in [9] includes the harmonic bus voltages at all buses as unknowns in such a system.

\subsection{Paper contribution}

This paper presents an improved HLF formulation which enhances the convergence properties of traditional HLF formulations without losing accuracy. The proposed formulation approaches the HLF problem as a single nonlinear equation system, considering the harmonic bus voltage influence on NLL behaviour but not including harmonic bus voltages at linear buses as unknowns. In order to tackle any electrical network operating under highly distorted bus voltages, two improvements are made over the HLF formulation described in $[10,11]$ : firstly, its generalization to any sort of nonlinear load, and secondly, its application by using the Newton-Raphson method with true Jacobian matrix to reduce the inherent increase in the number of iterations. A three-bus network operating under highly distorted bus voltages is solved to compare the performance of the proposed HLF formulation with the traditional formulations in the literature. 


\section{Harmonic load flow formulations}

HLF formulation is a modification of FLF formulation which includes harmonic bus voltages and NLL state variables [1]. Many HLF formulations in the literature look for a compromise between simplicity to reduce numerical convergence problems and reliability to provide accurate results. The next Subsections present the main formulations, from the simplest to the most complicated, when in electric power systems only the following types of buses are considered: Slack bus $(i=1)$, power consumption buses (PQ buses) $(i=2, \ldots, c)$ and buses feeding NLLs (NL buses) $(i=c+1, \ldots, n)$. The absence of PV buses in electric power systems is assumed without loss of generality. The usual assumption that power injection is mainly due to the fundamental bus voltage and current components, i.e., $\left.\underline{S}_{i} \approx \underline{V}_{i}^{1}{ }^{\cdot} \cdot \underline{I}_{i}{ }^{1}\right)^{*}$, is made. Even harmonics are not taken into account in the study as they are negligible due to the bus voltage and current half-wave symmetry.

\subsection{Harmonic penetration}

Harmonic penetration (HP) is the simplest frequency domain procedure [7]. HP is illustrated in Fig. 1 and its data and unknowns are summarised in Table 1. This formulation considers that NLL behaviour depends only on the fundamental bus voltages and their own state variables (i.e., no harmonic interaction is assumed in NLL behaviour). This allows NLL equations which define NLL behaviour to be incorporated into FLF. Thus, the nonlinear equation system of the modified FLF $\left(\mathrm{FLF}_{\mathrm{m}}\right)$ is derived from the FLF equations, where NLLs are treated as PQ loads by adding the NLL equations, i.e., 


$$
\begin{aligned}
& \underline{S}_{i}=\underline{V}_{i}^{1}\left(\sum_{j=1}^{n} \underline{Y}_{i j}^{1} \underline{V}_{j}^{1}\right)^{*} \quad(i=2, \ldots, c) \\
& \underline{V}_{i}^{1}\left(\underline{I}_{i}^{1}\right)^{*}=\underline{V}_{i}^{1}\left(\sum_{j=1}^{n} \underline{Y}_{i j}^{1} \underline{V}_{j}^{1}\right)^{*} \quad(i=c+1, \ldots, n) \\
& n l_{i}^{r}\left(\underline{V}_{i}^{1}, \delta_{i}^{r}, D_{i}^{m}\right)=0 \quad\left(i=c+1, \ldots, n ; r=1, \ldots, r_{\max } ; m=1, \ldots, m_{\max }\right),
\end{aligned}
$$

where $\underline{Y}_{i j}^{1}$ are the $i j^{\text {th }}$ elements of the fundamental bus admittance matrix $\mathbf{Y}^{1}$, and $n l^{r}{ }_{i}(\cdot)=0$ represents the NLL equations. These equations depend on $D^{m}{ }_{i}$ (NLL data) and $\delta^{r}{ }_{i}$ (NLL state variables). The NLL injected fundamental bus currents are expressed as

$$
\underline{I}_{i}^{1}=\underline{f}_{i}^{1}\left(\underline{V}_{i}^{1}, \delta_{i}^{r}, D_{i}^{m}\right) \quad\left(i=c+1, \ldots, n ; r=1, \ldots, r_{\max } ; m=1, \ldots, m_{\max }\right) .
$$

The numerical resolution of the equation system (1) provides the fundamental bus voltages $\underline{V}_{i}^{1}$ and the NLL state variables $\delta^{r}{ }_{i}$ which are used to obtain the harmonic bus voltages $\underline{V}_{i}^{h}$ by the voltage node (VN) method. This method is based on the resolution of the linear systems

$$
\mathbf{Y}_{\mathbf{B}}^{h} \cdot \mathbf{V}_{\mathbf{B}}^{h}=\mathbf{I}_{\mathbf{B}}^{h},
$$

where the NLL injected harmonic bus currents $\underline{I}_{i}^{h}(i=c+1, \ldots, n)$ and the harmonic bus admittances at the Slack and PQ buses $\underline{Y}_{i}^{h}(i=1, \ldots, c)$ must be incorporated into the injected harmonic bus current vectors $\mathbf{I}_{\mathbf{B}}^{h}$ and the harmonic bus admittance matrices $\mathbf{Y}_{\mathbf{B}}^{h}$, respectively, and are determined as

$$
\begin{aligned}
& \underline{I}_{i}^{h}=\underline{f}_{i}^{h}\left(\underline{V}_{i}^{1}, \delta_{i}^{r}, D_{i}^{m}\right) \quad\left(i=c+1, \ldots, n ; r=1, \ldots, r_{\max } ; m=1, \ldots, m_{\max }\right) \\
& \underline{Y}_{1}^{h}=\frac{1}{R_{1}+j \cdot h \cdot X_{1}^{1}} \quad ; \quad R_{1} \approx \frac{X_{1}^{1}}{20} \\
& \underline{Y}_{i}^{1}=\frac{-\underline{S}_{i}^{*}}{\left(V_{i}^{1}\right)^{2}}=\underline{f}_{Y i}^{1}\left(V_{i}^{1}\right) \quad ; \quad \underline{Y}_{i}^{h}=\frac{1}{\operatorname{Re}\left\{1 / \underline{Y}_{i}^{1}\right\}+j \cdot h \cdot \operatorname{Im}\left\{1 / \underline{Y}_{i}^{1}\right\}}=\underline{f}_{Y i}^{h}\left(V_{i}^{1}\right) \quad(i=2, \ldots, c) .
\end{aligned}
$$

It must be noted that an $X / R$ ratio equal to 20 is assumed in (4) for the Slack bus 
fundamental impedance.

This formulation allows the HLF problem to be tackled in a simple way, as with the FLF problem, but NLL sensitiveness to harmonic bus voltages could result in overestimation of the NLL polluting effect if harmonic bus voltage distortion is high.

\subsection{Simplified harmonic load flow}

Simplified harmonic load flow (SHLF) performs a fixed-point iteration method on a set of two nonlinear equation systems (labelled as $\mathrm{N}_{1}$ and $\mathrm{N}_{2}$ ) which are solved separately and in sequence by applying some iterative numerical method to each of them (see Fig. 1) [8]. SHLF data and unknowns are summarised in Table 1.

The first nonlinear equation system $\mathrm{N}_{1}$ is the $\mathrm{FLF}_{\mathrm{m}}$ in HP considering NLL harmonic interaction, i.e.,

$$
\begin{aligned}
& \underline{S}_{i}=\underline{V}_{i, \mathrm{~N}_{1}}^{1}\left(\sum_{j=1}^{n} \underline{Y}_{i j}^{1} \underline{V}_{j, \mathrm{~N}_{1}}^{1}\right)^{*} \quad(i=2, \ldots, c) \\
& \underline{V}_{i, \mathrm{~N}_{1}}^{1}\left(\underline{I}_{i}^{1}\right)^{*}=\underline{V}_{i, \mathrm{~N}_{1}}^{1}\left(\sum_{j=1}^{n} \underline{Y}_{i j}^{1} \underline{V}_{j, \mathrm{~N}_{1}}^{1}\right)^{*} \quad(i=c+1, \ldots, n) \\
& n l_{i}^{r}\left(\underline{V}_{i, \mathrm{~N}_{1}}^{1}, \underline{V}_{i, \mathrm{~N}_{2}}^{h}, \delta_{i, \mathrm{~N}_{\mathrm{l}}}^{r}, D_{i}^{m}\right)=0 \quad\left(i=c+1, \ldots, n ; r=1, \ldots, r_{\max } ; m=1, \ldots, m_{\max }\right),
\end{aligned}
$$

where the NLL injected fundamental bus currents are expressed as

$$
\underline{I}_{i}^{1}=\underline{f}_{i}^{1}\left(\underline{V}_{i, \mathrm{~N}_{1}}^{1}, \underline{V}_{i, \mathrm{~N}_{2}}^{h}, \delta_{i, \mathrm{~N}_{1}}^{r}, D_{i}^{m}\right) \quad\left(i=c+1, \ldots, n ; r=1, \ldots, r_{\max } ; m=1, \ldots, m_{\max }\right) .
$$

Note that, although harmonic interaction is assumed in NLL behaviour, the harmonic bus voltages $\underline{V}_{i, \mathrm{~N}_{2}}^{h}$ in the NLL functions $n l^{r}{ }_{i}(\cdot)=0$ and $\underline{I}_{i}{ }_{i}=f^{1}{ }_{i}(\cdot)$ are data from the second nonlinear equation system $\mathrm{N}_{2}$, where these variables are unknowns. Thus, the numerical resolution of (5) provides the fundamental bus voltages $\underline{V}_{i, \mathrm{~N} \text { i }}^{1}$ and the NLL state variables $\delta_{i, \mathrm{~N} 1}^{r}$ which are used in the second nonlinear equation system.

Once (5) is solved, the linear network with the Slack and PQ buses (linear buses) is represented by its generalised Thévenin equivalent circuits "observed" from the NL 
buses [8]. The second nonlinear equation system $\mathrm{N}_{2}$ is the HA and is built by considering as unknowns only those of the NL buses. Its equations are based on Kirchhoff's second law applied to the fundamental and harmonic Thévenin equivalent circuits, and the NLL equations which define NLL behaviour, i.e.,

$$
\begin{aligned}
& \underline{V}_{i, \mathrm{~N}_{2}}^{k}=\underline{E}_{T h i}^{k}+\sum_{j=c+1}^{n} \underline{Z}_{T h i j}^{k} \underline{I}_{i}^{k} \quad(i=c+1, \ldots, n) \\
& n l_{i}^{r}\left(\underline{V}_{i, \mathrm{~N}_{2}}^{1}, \underline{V}_{i, \mathrm{~N}_{2}}^{h}, \delta_{i, \mathrm{~N}_{2}}^{r}, D_{i}^{m}\right)=0 \quad\left(i=c+1, \ldots, n ; r=1, \ldots, r_{\max } ; m=1, \ldots, m_{\max }\right),
\end{aligned}
$$

where the NLL fundamental and harmonic Thévenin equivalent circuit parameters and injected currents are expressed as

$$
\begin{aligned}
& \underline{E}_{T h i}^{1}=\underline{f}_{E T h i}^{1}\left(\underline{V}_{l, \mathrm{~N}_{1}}^{1}, \underline{V}_{i, \mathrm{~N}_{2}}^{1}\right) ; \quad \underline{E}_{T h i}^{h}=0 ; \quad \underline{Z}_{T h i j}^{k}=\underline{f}_{Z T h i j}^{k}\left(V_{l, \mathrm{~N}_{1}}^{1}\right) \\
& (i=c+1, \ldots, n ; j=c+1, \ldots, n ; l=2, \ldots, c) \\
& \underline{I}_{i}^{k}=\underline{f}_{i}^{k}\left(\underline{V}_{i, \mathrm{~N}_{2}}^{1}, \underline{V}_{i, \mathrm{~N}_{2}}^{h}, \delta_{i, \mathrm{~N}_{2}}^{r}, D_{i}^{m}\right) \\
& \left(i=c+1, \ldots, n ; r=1, \ldots, r_{\max } ; m=1, \ldots, m_{\max }\right) .
\end{aligned}
$$

Note that the fundamental bus voltages at the PQ buses $\underline{V}_{l, \mathrm{~N} 1}^{1}$ in the Thévenin equivalent circuit functions $\underline{E}_{T h i}^{1}=f_{E T h i}^{1}(\cdot)$ and $\underline{Z}_{T h i j}^{k}=f_{Z T h i j}^{k}(\cdot)$ are data from the first nonlinear equation system $\mathrm{N}_{1}$, where these variables are unknowns. The numerical resolution of (7) provides the fundamental and harmonic bus voltages at the NL buses $\underline{V}_{i, \mathrm{~N} 2}^{k}$ and the NLL state variables $\delta_{i, \mathrm{~N} 2}^{r}$. Convergence of the fixed-point iteration algorithm is checked by comparing the fundamental bus voltages at the NL buses obtained from the first and second nonlinear equation systems (i.e., $\underline{V}_{i, \mathrm{~N} 1}^{1}$ and $\underline{V}_{i, \mathrm{~N} 2}^{1}$ ). If convergence is reached, the VN method (3) is applied to determine the harmonic bus voltages at the Slack and PQ buses $\underline{V}_{i}^{h}$. Otherwise, a new fixed-point iteration is made by using the results of (7) in the first nonlinear equation system.

This formulation takes into consideration the harmonic bus voltage influence on NLL behaviour without introducing the harmonic bus voltages at the Slack and PQ buses as unknowns of the nonlinear equations. However, it could pose convergence 
problems depending on the degree of decoupling between the two nonlinear equation systems (5) and (7) (see Section 3).

\subsection{Complete harmonic load flow}

Complete harmonic load flow (CHLF) is a natural modification of FLF where NLL treatment and harmonic bus voltage calculation are included considering harmonic interaction in NLL behaviour. CHLF data and unknowns are summarised in Table 1. CHLF is based on the simultaneous resolution of power equations at the PQ buses and harmonic bus current balance at the Slack and PQ buses, together with fundamental and harmonic bus current balance and NLL equations at the NL buses (see Fig. 1) [9], i.e.,

$$
\begin{aligned}
& \underline{S}_{i}=\underline{V}_{i}^{1}\left(\sum_{j=1}^{n} \underline{Y}_{i j}^{1} \underline{V}_{j}^{1}\right)^{*} \quad(i=2, \ldots, c) \\
& -\underline{Y}_{i}^{h} \underline{V}_{i}^{h}=\sum_{j=1}^{n} \underline{Y}_{i j}^{h} \underline{V}_{j}^{h} \quad(i=1, \ldots, c) \\
& \underline{I}_{i}^{k}=\sum_{j=1}^{n} \underline{Y}_{i j}^{k} \underline{V}_{j}^{k} \quad(i=c+1, \ldots, n) \\
& n l_{i}^{r}\left(\underline{V}_{i}^{1}, \underline{V}_{i}^{h}, \delta_{i}^{r}, D_{i}^{m}\right)=0 \quad\left(i=c+1, \ldots, n ; r=1, \ldots, r_{\max } ; m=1, \ldots, m_{\max }\right),
\end{aligned}
$$

where $\underline{Y}_{i j}^{k}$ are the $i j^{\text {th }}$ elements of the fundamental and harmonic bus admittance matrices $\mathbf{Y}_{\mathbf{B}}^{k}$, and $\underline{Y}_{i}^{h}$ are the harmonic bus admittances at the Slack and PQ buses (4). The NLL injected fundamental and harmonic bus currents are expressed as

$$
\underline{I}_{i}^{k}=\underline{f}_{i}^{k}\left(\underline{V}_{i}^{1}, \underline{V}_{i}^{h}, \delta_{i}^{r}, D_{i}^{m}\right) \quad\left(i=c+1, \ldots, n ; r=1, \ldots, r_{\max } ; m=1, \ldots, m_{\max }\right) .
$$

This formulation allows the HLF problem to be tackled as a single nonlinear equation system where the harmonic bus voltages at the Slack and PQ buses are also included as unknowns. This increases the number of unknowns to be determined significantly, which can result in degradation of the convergence properties characterising numerical resolution methods. 


\section{Convergence analysis of numerical resolution methods}

HLF calculation can be regarded as the resolution of a nonlinear equation system formulated as a set of $q$ equations in $q$ unknowns $F(x)=0$ :

$$
\left\{f_{i}\left(x_{1}, x_{2}, \ldots, x_{q}\right)=0 \quad(i=1, \ldots, q) .\right.
$$

The numerical resolution of this system provides the fundamental and harmonic bus voltages and the NLL state variables. Multiple solutions are mathematically possible for the above nonlinear equation system, but usually only one is physically admissible. Several numerical methods can solve this system, among which NewtonRaphson is the most widely used in the bibliography. Fixed-point iteration methods, such as the Gauss-Seidel method, can also be used but have poorer convergence properties. The SHLF formulation is an example of these iterative approaches.

\subsection{Newton-Raphson method}

The Newton-Raphson method can be algorithmically expressed as

$$
x^{(\alpha+1)}=x^{(\alpha)}-D F^{-1}\left(x^{(\alpha)}\right) \cdot F\left(x^{(\alpha)}\right),
$$

which is applied from the initial value $x^{(0)}$ to the problem solution $x^{(\mathrm{S})}$, with $D F(x)$ being the Jacobian matrix of the nonlinear equation system and $\alpha+1$ the NewtonRaphson iteration. Jacobian matrix is called true Jacobian matrix if calculated for each of the iterations. It would be called constant Jacobian matrix if it were computed for only the very first iteration and remained unchanged for the rest of iterations. Algorithm convergence can be checked from the conditions $\left\|F\left(x^{(\alpha+1)}\right)\right\|<\varepsilon$ or $\left\|x^{(\alpha+1)}-x^{(\alpha)}\right\|<\varepsilon$, where $\varepsilon$ is a fixed error. The main drawback of this method is that convergence to the problem solution is only assured if the initial value is close to the solution and $\operatorname{det}\left(D F\left(x^{(\alpha)}\right)\right) \neq 0$ for all $x^{(\alpha)}$. Otherwise (more likely in HLF than in FLF 
due to initial values of harmonic bus voltages far from the solution), it can lead to divergence or an unfeasible solution. This is illustrated in Fig. 2(a). If the initial value is $x^{(01)}$, there exists a value $x^{(\alpha)}$ where $\operatorname{det}\left(D F\left(x^{(\alpha)}\right)\right)=0$, and this may cause divergence. When the initial value is $x^{(02)}$, convergence to the feasible solution $x^{(\mathrm{FS})}$ may occur because $x^{(02)}$ is close to $x^{(\mathrm{FS})}$ and $\operatorname{det}\left(D F\left(x^{(\alpha)}\right)\right) \neq 0$ for all $x^{(\alpha)}$. However, if the initial value is $x^{(03)}$, convergence to an unfeasible solution $x^{(\mathrm{US})}$ may occur because $x^{(03)}$ is closer to $x^{(\mathrm{US})}$ than to $x^{(\mathrm{FS})}$.

\subsection{Fixed-point iteration method}

The nonlinear equation system $F(x)=0$ can occasionally be reformulated as a set of $q$ equations in $q$ unknowns $x=G(x)$ :

$$
\left\{x_{i}=g_{i}\left(x_{1}, x_{2}, \ldots, x_{q}\right) \quad(i=1, \ldots, q) .\right.
$$

Most fixed-point iteration methods can be algorithmically expressed as

$$
x^{(\beta+1)}=G\left(x^{(\beta)}\right),
$$

which is applied from the initial value $x^{(0)}$ to the problem solution $x^{(\mathrm{S})}$, with $G(x)$ being a nonlinear equation system derived from $F(x)$ and $\beta+1$ the fixed-point iteration.

Algorithm convergence is checked from the condition $\left\|x^{(\beta+1)}-x^{(\beta)}\right\|<\varepsilon$, where $\varepsilon$ is a fixed error. The main drawback of this method is that convergence to the problem solution is only assured if the initial value is close to the solution and $\left\|D G\left(x^{(\mathrm{S})}\right)\right\|<1$. Otherwise, it can lead to divergence or an unfeasible solution. This is illustrated in Fig. 2(b). If the initial value is $x^{(01)}$, then $\left\|D G_{+}\left(x^{(\mathrm{FS})}\right)\right\|>1$, and this causes divergence. When the initial value is $x^{(02)}$, convergence to the feasible solution $x^{(\mathrm{FS})}$ occurs because $x^{(02)}$ is close to $x^{(\mathrm{FS})}$ and $\left\|D G_{-}\left(x^{(\mathrm{FS})}\right)\right\|<1$. However, if the initial value is $x^{(03)}$, convergence to an unfeasible solution $x^{(\mathrm{US})}$ occurs because $x^{(03)}$ is closer to $x^{(\mathrm{US})}$ than 
to $x^{(\mathrm{FS})}$.

One of the main fixed-point iteration methods is the Gauss-Seidel method, whose associated algorithm is

$$
\left\{x_{i}^{(\beta+1)}=g_{i}\left(x_{1}^{(\beta+1)}, \ldots, x_{i-1}^{(\beta+1)}, x_{i}^{(\beta)}, \ldots, x_{q}^{(\beta)}\right) \quad(i=1, \ldots, q) .\right.
$$

At each fixed-point iteration $\beta+1$, the equations of (15) are used separately and in sequence. Thus, $g_{1}(\cdot)$ allows $x_{1}^{(\beta+1)}$ to be obtained, $g_{2}(\cdot)$ allows $x_{2}^{(\beta+1)}$ to be obtained, and so on.

If it is not possible to reformulate the nonlinear equation system $F(x)=0$ as $x=$ $G(x)$, the previous algorithm must be rewritten as follows:

$$
\left\{f_{i}\left(x_{1}^{(\beta+1)}, \ldots, x_{i}^{(\beta+1)}, x_{i+1}^{(\beta)}, \ldots, x_{q}^{(\beta)}\right)=0 \quad(i=1, \ldots, q) .\right.
$$

At each fixed-point iteration $\beta+1$, the equations of (16) are solved separately and in sequence by applying some iterative numerical method to each of them. Thus, the numerical resolution of $f_{1}(\cdot)=0$ allows $x_{1}^{(\beta+1)}$ to be obtained, the numerical resolution of $f_{2}(\cdot)=0$ allows $x_{2}^{(\beta+1)}$ to be obtained, and so on.

\subsection{SHLF fixed-point iteration method}

The fixed-point iteration method of SHLF formulation can be regarded as the application of the Gauss-Seidel method to a set of two nonlinear equation systems:

$$
\left\{\begin{array}{l}
F_{\mathrm{N}_{1}}(u, v, w)=0 \\
F_{\mathrm{N}_{2}}(u, v, w)=0
\end{array} .\right.
$$

The Gauss-Seidel method is applied to (17) by using the following algorithm:

$$
\left\{\begin{array}{c}
F_{\mathrm{N}_{1}}\left(u_{\mathrm{N}_{1}}^{(\beta+1)}, v^{(\beta+1)}, w^{(\beta)}\right)=0 \\
F_{\mathrm{N}_{2}}\left(u_{\mathrm{N}_{2}}^{(\beta+1)}, v^{(\beta+1)}, w^{(\beta+1)}\right)=0
\end{array} .\right.
$$


At each fixed-point iteration $\beta+1$, the nonlinear equation systems of (18) are solved separately and in sequence by applying the Newton-Raphson method to each of them (see Fig. 2(c)). Thus, the numerical resolution of $F_{\mathrm{N}_{1}}(\cdot)=0$ allows $v^{(\beta+1)}\left(\right.$ and $\left.u_{\mathrm{N}_{1}}{ }^{(\beta+1)}\right)$ to be obtained by applying the iterative scheme

$$
\left\{\begin{array}{l}
x_{\mathrm{N}_{1}}=\left[\begin{array}{ll}
u & v
\end{array}\right]^{T} ;\left(x_{\mathrm{N}_{1}}^{(\beta+1)}\right)^{(0)}=\left[\left(u_{\mathrm{N}_{2}}^{(\beta)}\right)^{(\mathrm{S})}\left(v^{(\beta)}\right)^{(\mathrm{S})}\right]^{T} \\
\left(x_{\mathrm{N}_{1}}^{(\beta+1)}\right)^{(\alpha+1)}=\left(x_{\mathrm{N}_{1}}^{(\beta+1)}\right)^{(\alpha)}-D \bar{F}_{\mathrm{N}_{1}}^{-1}\left(\left(x_{\mathrm{N}_{1}}^{(\beta+1)}\right)^{(\alpha)}\right) \bar{F}_{\mathrm{N}_{1}}\left(\left(x_{\mathrm{N}_{1}}^{(\beta+1)}\right)^{(\alpha)}\right)^{\prime}
\end{array}\right.
$$

the numerical resolution of $F_{\mathrm{N}_{2}}(\cdot)=0$ allows $w^{(\beta+1)}\left(\right.$ and $\left.u_{\mathrm{N} 2}{ }^{(\beta+1)}\right)$ to be obtained by applying the iterative scheme

$$
\left\{\begin{array}{l}
x_{\mathrm{N}_{2}}=\left[\begin{array}{ll}
u & w
\end{array}\right]^{T} ;\left(x_{\mathrm{N}_{2}}^{(\beta+1)}\right)^{(0)}=\left[\left(u_{\mathrm{N}_{1}}^{(\beta+1)}\right)^{(\mathrm{S})}\left(w^{(\beta)}\right)^{(\mathrm{S})}\right]^{T} \\
\left(x_{\mathrm{N}_{2}}^{(\beta+1)}\right)^{(\alpha+1)}=\left(x_{\mathrm{N}_{2}}^{(\beta+1)}\right)^{(\alpha)}-D \bar{F}_{\mathrm{N}_{2}}^{-1}\left(\left(x_{\mathrm{N}_{2}}^{(\beta+1)}\right)^{(\alpha)}\right) \bar{F}_{\mathrm{N}_{2}}\left(\left(x_{\mathrm{N}_{2}}^{(\beta+1)}\right)^{(\alpha)}\right)^{\prime}
\end{array}\right.
$$

and so on.

Subsection 2.2 identifies the unknowns $u_{\mathrm{N} 1}, u_{\mathrm{N} 2}, v$ and $w$ with those of SHLF formulation:

$$
\begin{aligned}
& u_{\mathrm{N}_{1}}=\left(u_{\mathrm{I}, \mathrm{N}_{1}}, u_{\mathrm{II}, \mathrm{N}_{1}}\right)=\left(\underline{V}_{i, \mathrm{~N}_{1}}^{1}, \delta_{i, \mathrm{~N}_{1}}^{r}\right) \quad\left(i=c+1, \ldots, n ; r=1, \ldots, r_{\max }\right) \\
& u_{\mathrm{N}_{2}}=\left(u_{\mathrm{I}, \mathrm{N}_{2}}, u_{\mathrm{II}, \mathrm{N}_{2}}\right)=\left(\underline{V}_{i, \mathrm{~N}_{2}}^{1}, \delta_{i, \mathrm{~N}_{2}}^{r}\right) \quad\left(i=c+1, \ldots, n ; r=1, \ldots, r_{\max }\right) \\
& v=\underline{V}_{i, \mathrm{~N}_{1}}^{1} \quad(i=2, \ldots, c) \\
& w=\underline{V}_{i, \mathrm{~N}_{2}}^{h} \quad(i=c+1, \ldots, n) .
\end{aligned}
$$

Instead of the usual condition to check fixed-point iteration algorithm convergence

$$
\left\|\left(v^{(\beta+1)}, w^{(\beta+1)}\right)-\left(v^{(\beta)}, w^{(\beta)}\right)\right\|<\varepsilon,
$$

where $\varepsilon$ is a fixed error, an alternative condition is used to check SHLF fixed-point iteration algorithm convergence:

$$
\left\|u_{\mathrm{I}, \mathrm{N}_{2}}^{(\beta+1)}-u_{\mathrm{I}, \mathrm{N}_{1}}^{(\beta+1)}\right\|<\varepsilon
$$


Convergence problems associated with the SHLF fixed-point iteration method are similar to those of the fixed-point iteration method described in Subsection 3.2.

\section{An improved harmonic load flow formulation}

The proposed HLF formulation, known as improved unified harmonic load flow (IUHLF), derives from the unified harmonic load flow (UHLF) [10, 11]. Both formulations are a modification of the CHLF $\left(\mathrm{CHLF}_{\mathrm{m}}\right)$ which takes into account the Thévenin equivalent circuit approach used in SHLF to avoid considering the harmonic bus voltages at the Slack and PQ buses as unknowns. These formulations are illustrated in Fig. 1 and their data and unknowns are summarised in Table 1.

\subsection{Unified harmonic load flow and improved unified harmonic load flow}

UHLF formulation, $[10,11]$, is based on the simultaneous resolution of the power equations at the PQ buses, Kirchhoff's second law applied to the fundamental and harmonic Thévenin equivalent circuits, and the NLL equations defining HVDC converter behaviour, i.e.,

$$
\begin{aligned}
& \underline{S}_{i}=\underline{V}_{i}^{1}\left(\sum_{j=1}^{n} \underline{Y}_{i j}^{1} \underline{V}_{j}^{1}\right)^{*} \quad(i=2, \ldots, c) \\
& \underline{V}_{i}^{k}=\underline{E}_{T h i}^{k}+\sum_{j=c+1}^{n} \underline{Z}_{T h i j}^{k} \underline{I}_{i}^{k} \quad(i=c+1, \ldots, n) \\
& h v d c_{i}^{r}\left(\underline{V}_{i}^{1}, \underline{V}_{i}^{h}, \delta_{i}^{r}, D_{i}^{m}\right)=0 \quad\left(i=c+1, \ldots, n ; r=1, \ldots, r_{\max } ; m=1, \ldots, m_{\max }\right),
\end{aligned}
$$

where $\underline{Y}^{1}{ }_{i j}$ are the $i j^{\text {th }}$ elements of the fundamental bus admittance matrix $\mathbf{Y}^{1}{ }_{\mathbf{B}}, \underline{I}_{i}^{k}$ is defined in (10), and $h v d c^{r}{ }_{i}(\cdot)=0$ represents the HVDC converter equations.

The fundamental and harmonic Thévenin equivalent circuits of the linear network “observed" from the NL buses (i.e., $\underline{E}_{T h i}^{k}$ and $\underline{Z}_{T h i j}^{k}$ ) are determined in each of the iterations to be used in the corresponding equations since $\underline{E}_{T h i}^{1}=f_{E T h i}^{1}\left(\underline{V}_{l}^{1}, \underline{V}_{i}^{1}\right)$, $\underline{E}^{h}{ }_{T h i}=0$ and $\underline{Z}_{T h i j}^{k}=f^{k}{ }_{Z T h i j}\left(V^{1}\right)(l=2, \ldots, c)$. The use of a constant Jacobian matrix is 
proposed in [10] to carry out the numerical resolution of (24) by the Newton-Raphson method. Holding the Jacobian matrix constant leads to a larger number of faster iterations to obtain the overall solution, [11]. However, if bus voltages are highly distorted, this number of iterations may still be larger or convergence to the solution might even not be achieved. The numerical resolution of (24) provides the fundamental bus voltages at the PQ and NL buses, the harmonic bus voltages at the NL buses and the NLL state variables. Subsequently, the VN method (3) is applied to determine the harmonic bus voltages at the Slack and PQ buses.

This formulation allows the HLF problem to be tackled as a single nonlinear equation system, considering NLL harmonic interaction but not including the harmonic bus voltages at the Slack and PQ buses as unknowns.

Another UHLF formulation could be considered, namely removing the following equations of Kirchhoff's second law applied to the fundamental Thévenin equivalent circuits from (24):

$$
\underline{V}_{i}^{1}=\underline{E}_{T h i}^{1}+\sum_{j=c+1}^{n} \underline{Z}_{T h i j}^{1} \underline{I}_{i}^{1} \quad(i=c+1, \ldots, n)
$$

and incorporating the following power equations derived from HVDC converters when treated as PQ loads into (24) instead:

$$
\underline{V}_{i}^{1}\left(\underline{I}_{i}^{1}\right)^{*}=\underline{V}_{i}^{1}\left(\sum_{j=1}^{n} \underline{Y}_{i j}^{1} \underline{V}_{j}^{1}\right)^{*} \quad(i=c+1, \ldots, n)
$$

However, this alternative UHLF formulation is discarded due to the high degree of nonlinearity of equations in (26) compared to those in (25).

Two features of the considered UHLF formulation are as follows [10, 11]:

- The formulation is oriented to the presence of a specific sort of nonlinear load in the electrical network: HVDC converters. 
- The Newton-Raphson method with constant Jacobian matrix is applied for the numerical resolution of (24).

While these two features could be good enough for electrical networks with the presence of HVDC converters and in a context of scarcely distorted bus voltages, [10, 11], they could not be convenient for electrical networks with the presence of any sort of nonlinear load or in scenarios with highly distorted bus voltages.

To overcome these limitations, an enhanced UHLF formulation called IUHLF formulation is presented. The two improvements over the UHLF formulation are as follows:

- The proposed formulation is oriented to the presence of any sort of nonlinear load in the electrical network. Therefore, $h v d c^{r}{ }_{i}(\cdot)=0$ in (24) must be replaced by $n l^{r}{ }_{i}(\cdot)=0$.

- The Newton-Raphson method with true Jacobian matrix is applied for the numerical resolution of (24). It allows the increase in the number of iterations which is inherent to the presence of highly distorted bus voltages in electrical networks to be smaller.

\subsection{Discussion on IUHLF formulation strengths}

The main IUHLF formulation strengths over other HLF formulations for electrical networks operating under highly distorted bus voltages are:

- In contrast to HP formulation, NLL harmonic interaction is considered in IUHLF formulation.

- The numerical resolution of IUHLF formulation is carried out by applying the Newton-Raphson method to a single nonlinear equation system, whereas the numerical resolution of SHLF formulation requires the application of the GaussSeidel method to a set of two nonlinear equation systems. The application of a 
fixed-point iteration method to the set of two systems in SHLF formulation hinders the global numerical resolution of the HLF problem, to such a point that high harmonic distortions might lead to very different values of $u_{\mathrm{N}_{1}}$ and $u_{\mathrm{N} 2}$. Therefore, convergence of SHLF formulation is improved by IUHLF formulation.

- The number of unknowns at $\mathrm{CHLF}_{\mathrm{m}}$ stage in IUHLF formulation is smaller than in CHLF formulation (the harmonic bus voltages at the Slack and PQ buses are not unknowns at that stage). Thus, convergence of IUHLF formulation is likely better than that of CHLF formulation.

- The use of Newton-Raphson method with true Jacobian matrix allows convergence of UHLF formulation to be enhanced by IUHLF formulation.

- Unlike UHLF formulation, IUHLF formulation is applicable to electrical networks with the presence of any kind of nonlinear load.

- IUHLF formulation exhibits the same accuracy as SHLF, CHLF and UHLF formulations (and better accuracy than HP formulation) because of the NLL harmonic interaction consideration in all four.

\section{Application}

The HLF problem associated with the three-bus network in Fig. 3(a) is studied. Typical NLLs, specifically single-phase uncontrolled rectifiers, connected to bus 3 , and an increasing number $N$ of these NLLs at the bus, are considered. The circuit of a single-phase uncontrolled rectifier [16] is shown in Fig. 3(b) and its supply voltage $v$, ac consumed current $i$ and dc voltage waveforms are depicted in Fig. 3(c), where $\omega=2 \pi \cdot f$ and $f$ is the fundamental frequency of the supply system. A distorted supply voltage $v$ is considered in the rectifier model to allow harmonic interaction to be assumed in NLL behaviour. The commutation angles $\theta_{i}$ are the NLL state variables, 
whose values must be determined irrespective of HLF formulation, i.e., they are always unknowns in the HLF resolution. The half-wave symmetry hypothesis is considered; therefore, only $\theta_{1}$ and $\theta_{2}$ must be determined since the commutation angles verify the relation $\theta_{j+2}=\theta_{j}+\pi(j=1,2)$. The data of the whole network are summarised in Table 2.

The proposed HLF problem is solved by using the HLF formulations in Section 2 (HP, SHLF and CHLF) and the improved HLF formulation in Section 4 (IUHLF) so as to compare their performances (UHLF formulation is not used due to the presence of NLLs which are not HVDC converters). The behaviour of the different formulations in the three-bus network operating under highly distorted bus voltages is analysed. In addition, the use of Newton-Raphson method with constant Jacobian matrix in IUHLF formulation is studied through the same network and in the same context of bus voltage distortion.

\subsection{Comparison of HLF formulation convergences}

From a common initial value $x^{(0)}$ for all used formulations, Newton-Raphson convergence was checked from the condition $\left\|x^{(\alpha+1)}-x^{(\alpha)}\right\|<10^{-4}$, and the terms of the Jacobian matrix were calculated by the finite difference approach (the only feasible choice for large, complicated networks). SHLF fixed-point iteration convergence was checked by considering $\varepsilon=10^{-5}$ in (23).

Fig. 4 shows the evolution of $\left\|x^{(\alpha+1)}-x^{(\alpha)}\right\|$ versus the Newton-Raphson iteration $(\alpha+1)$ for all used formulations and four different numbers $N$ of single-phase uncontrolled rectifiers connected to bus 3. In SHLF formulation, evolutions associated with the $\mathrm{FLF}_{\mathrm{m}}$ and HA stages are plotted in sequence. It can be noticed that greater values of $N$ generally lead to a higher number of iterations to the problem solution. 
This increase in the number of iterations is especially pronounced for CHLF formulation, coherently exceeding for all values of $N$ what is expected in a context of scarcely distorted bus voltages.

It is also observed that HP and IUHLF formulations require a smaller number of iterations to the problem solution irrespective of $N$. Therefore, it can be concluded that both formulations are the best in terms of convergence.

Regarding the use of Newton-Raphson method with constant Jacobian matrix in IUHLF formulation, it has been checked that it leads to serious convergence problems. Consequently, this strategy should be avoided in scenarios with highly distorted bus voltages.

\subsection{Comparison of HLF formulation accuracies}

Regarding the voltage at bus 3, Fig. 5 plots the values of individual Harmonic Distortions (HDs) versus the harmonic order $h$, as well as the value of Total Harmonic Distortion (THD), for all used formulations and two different numbers $N$ of single-phase uncontrolled rectifiers connected to this bus. Higher values of $N$ lead to greater discrepancy between the results obtained from the HP formulation and those obtained from the other three formulations. This is because NLL harmonic interaction is not considered in HP formulation, rendering the other three formulations better in terms of accuracy. The high values of individual $H D$ at $h=15$ for all used formulations can be explained by the existence of a parallel resonance near $h=15$ in the equivalent circuit of the network "observed" from bus 3.

\subsection{HLF formulation convergences and accuracies in large systems}

The two numerical resolution methods on which the HLF formulations considered in this paper are based, i.e. Newton-Raphson method and fixed-point iteration (Gauss- 
Seidel) method, are analysed in Section 3. Reference [17] presents a comparative study of both methods for load flow analysis performed on five different standard IEEE test systems, namely IEEE 3-bus, 5-bus, 14-bus, 30-bus and 57-bus test systems. This study shows that, when the Gauss-Seidel method is applied, the number of iterations needed to converge increases with system size (number of buses). On the other hand, when the Newton-Raphson method is used, the number of iterations needed to converge remains relatively constant regardless of system size.

HP, CHLF and IUHLF formulations are based on the Newton-Raphson method, whereas SHLF formulation relies on a combination of the fixed-point iteration (Gauss-Seidel) method and the Newton-Raphson method. Therefore, according to the findings in [17], the conclusion in Subsection 5.1 can also be extended to large systems in terms of convergence (IUHLF and HP formulations are the best). In addition, as NLL harmonic interaction is not considered in HP formulation, the conclusion in Subsection 5.2 can also be extended to large systems in terms of accuracy (IUHLF, SHLF and CHLF formulations are better than HP formulation).

Consequently, although a larger, more complicated network than that in Fig. 3(a) could have been chosen to perform the analyses in Section 5, the selected network allows the conclusions obtained in Subsections 5.1 and 5.2 to be general. Thus, also for large systems, it is demonstrated that IUHLF formulation outperforms the other HLF formulations in terms of convergence and accuracy.

\section{Conclusions}

The paper presented and compared an improved formulation for the HLF problem with the most common formulations in the literature. The main findings are as follows. First, it is possible to propose an enhanced formulation based on the best properties of HP, SHLF, CHLF and UHLF formulations, namely the use of harmonic 
equivalent circuits to reduce the number of harmonic bus voltages included as unknowns in nonlinear equation systems, and the construction of a single nonlinear equation system numerically solved by the Newton-Raphson method with true Jacobian matrix. Second, the number of iterations required by the proposed formulation (IUHLF) is smaller than those of the other formulations with which it shares the same accuracy of results (SHLF, CHLF and UHLF), thus simplifying the numerical resolution of the HLF problem. This is particularly critical in scenarios with highly distorted bus voltages.

The following research lines are proposed as future work:

- Convergence properties for two numerical methods, i.e. the Newton-Raphson method and the fixed-point iteration method, were explored in the paper. There are many other numerical methods in the literature which can be considered for the numerical resolution of the HLF problem.

- During the numerical resolution of the HLF problem, it was observed that the initial value $x^{(0)}$ plays a significant role in finding the correct solution $x^{(\mathrm{S})}$ to the problem. There is a need to develop a more robust technique which gives a good estimate of the initial value $x^{(0)}$ for any type of HLF problem.

- An in-depth study of the HLF problem in electrical networks operating under highly distorted bus voltages and an investigation of specific numerical methods which might be helpful in the numerical resolution of such networks are suggested.

- Only a specific sort of nonlinear load was considered in this paper, i.e. the singlephase uncontrolled rectifier. The HLF problem could be further investigated for other important nonlinear loads such as discharge lamps, LED lamps, battery chargers, three-phase uncontrolled rectifiers, etc.

- An analysis of the HLF problem in a context of renewable energy resources, which 
entails the harmonic characterization of the renewable energy resources and the

study of their harmonic interaction with the electrical network, is recommended.

\section{References}

[1] Task Force on Harmonics Modeling and Simulation, "IEEE tutorial course on harmonics modeling and simulation," IEEE Power Eng. Soc., Jun. 2007, course text 07TP184.

[2] Kuo Lung Lian, and Taku Noda, "A time-domain harmonic power-flow algorithm for obtaining nonsinusoidal steady-state solutions," IEEE Trans. on Power Delivery, vol. 25, no. 3, pp. 1888-1898, Jul. 2010.

[3] D. K. Ray, S. Chattopadhyay, and S. Sengupta, "Estimation of harmonics on load flow in a multi-bus power system," Proceedings of the 1st IEEE International Conference on Control, Instrumentation, Energy and Communication (CIEC), pp. 472-475, Calcutta, India, 31 Jan.-2 Feb. 2014.

[4] A. L. Amoo, A. S. Bappah, and U. O. Aliyu, "Virtual platforms for teaching harmonic load flow to engineering students," Proceedings of the 1st IEEE International Conference on Emerging and Sustainable Technologies for Power and ICT in a Developing Society (NIGERCON), pp. 333-339, Owerri, Nigeria, 14-16 Nov. 2013.

[5] G. Constante-Flores, G. Cabrera-Celi, F. L. Quilumba, J. Játiva-Ibarra, and W.J. Lee, "A harmonic power flow program aimed at analyzing distortion effects caused by industrial customers," Proceedings of the 50th IEEE Industry Applications Society Annual Meeting (IAS), pp. 1-8, Addison, TX, 18-22 Oct. 2015.

[6] B. Poudel, and V. Cecchi, "Harmonic power flow using multi-segment frequency-dependent transmission line models," Proceedings of the 45th IEEE North American Power Symposium (NAPS), pp. 1-6, Manhattan, KS, 22-24 Sept. 2013.

[7] A. A. Mahmoud, and R. D. Shultz, "A method for analyzing harmonic distribution in A.C. power systems," IEEE Trans. on Power Apparatus and Systems, vol. PAS-101, no. 6, pp. 1815-1824, Jun. 1982.

[8] M. Valcárcel, and J. G. Mayordomo, "Harmonic power flow for unbalanced systems," IEEE Trans. on Power Delivery, vol. 8, no. 4, pp. 2052-2059, Oct. 1993.

[9] D. Xia, and G. T. Heydt, "Harmonic power flow studies-Part I and Part II," IEEE Trans. on Power Apparatus and Systems, vol. PAS-101, no. 6, pp. 12571265 and pp. 1266-1270, Jun. 1982.

[10] B. C. Smith, and J. Arrillaga, "Power flow constrained harmonic analysis in AC-DC power systems," IEEE Trans. on Power Systems, vol. 14, no. 4, pp. 1251-1261, Nov. 1999.

[11] J. Arrillaga, and B. Smith, AC-DC Power System Analysis. IEE Power and Energy Series, 27. London, UK: The Institution of Electrical Engineers, 1998.

[12] M. A. Moreno, and J. Usaola, "Three-phase harmonic load flow in frequency and time domains," IEE Proc. - Electric Power Applications, vol. 150, no. 3, pp. 295-300, May 2003.

[13] C. A. Canesin, L. C. O. de Oliveira, J. B. Souza, D. de O. de Lima, and R. P. Buratti, "A time-domain harmonic power-flow analysis in electrical energy 
distribution networks, using Norton models for non-linear loading," Proceedings of the 16th IEEE International Conference on Harmonics and Quality of Power (ICHQP), pp. 778-782, Bucharest, Romania, 25-28 May 2014.

[14] M. R. Irving, and M. J. H. Sterling, "Efficient Newton-Raphson algorithm for load-flow calculation in transmission and distribution networks," IEE Proc. CGeneration, Transmission and Distribution, vol. 134, no. 5, pp. 325-328, Sep. 1987.

[15] A. Keyhani, A. Abur, and S. Hao, "Evaluation of power flow techniques for personal computers," IEEE Trans. on Power Systems, vol. 4, no. 2, pp. 817-826, May 1989.

[16] J. J. Mesas, L. Sainz, and J. Molina, "Parameter estimation procedure for models of single-phase uncontrolled rectifiers," IEEE Trans. on Power Delivery, vol. 26, no. 3, pp. 1911-1919, Jul. 2011.

[17] H. Abaali, T. Talbi, and R. Skouri, "Comparison of Newton Raphson and Gauss Seidel methods for power flow analysis," World Academy of Science, Engineering and Technology, Open Science Index, Energy and Power Engineering, vol. 12, no. 9, pp. 627-633, Sep. 2018. Available at https://waset.org/publications/10009525

\section{Captions}

Figure 1. HLF formulation flowcharts $(h=3,5, \ldots ; k=1,3,5, \ldots)$.

Figure 2. Numerical methods: (a) Newton-Raphson method. (b) Fixed-point iteration method. (c) Iteration $\beta+1$ of SHLF fixed-point iteration method.

Figure 3. (a) Three-bus network. (b) Single-phase uncontrolled rectifier circuit. (c) Supply voltage $v$, ac current $i$ and dc voltage $v_{C}$ waveforms.

Figure 4. Evolution of $\left\|x^{(\alpha+1)}-x^{(\alpha)}\right\|$ versus the Newton-Raphson iteration $(\alpha+1)$ for all used formulations and four different values of $N$.

Figure 5. Voltage at bus 3: Individual $H D$ s versus the harmonic order $h$, as well as $T H D$, for all used formulations and two different values of $N$.

Table 1. HLF formulations.

Table 2. Network data. 
Figures

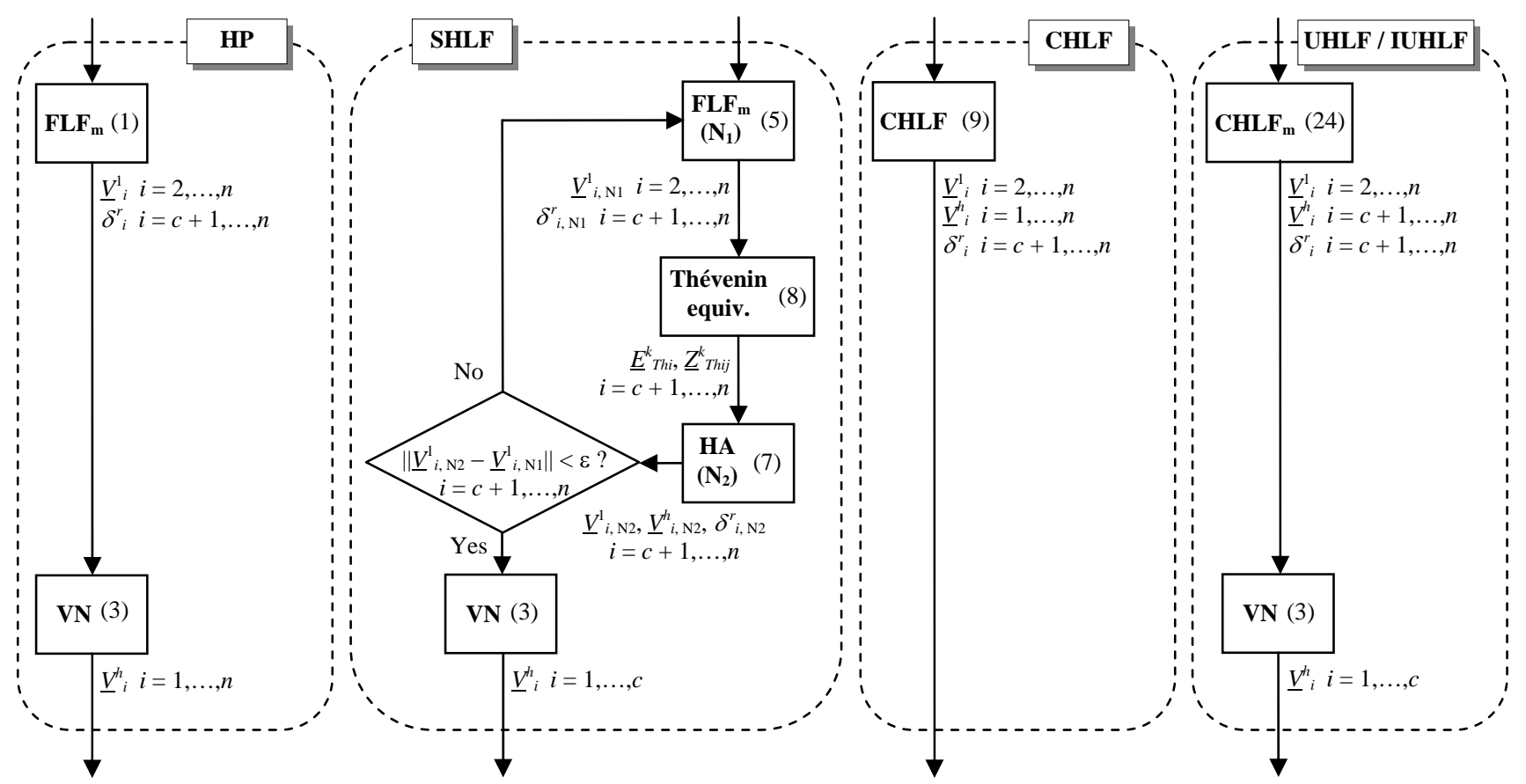

Figure 1. HLF formulation flowcharts $(h=3,5, \ldots ; k=1,3,5, \ldots)$. 
$x^{(\mathrm{FS})}$ : Feasible Solution $\quad x^{(\mathrm{US})}$ : Unfeasible Solution

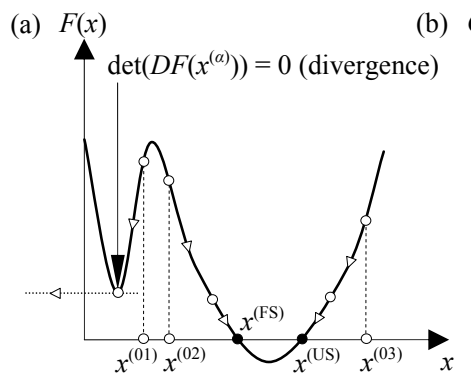

(b) $\left.G(x)\left\|D G_{+}\left(x^{(\mathrm{FS})}\right)\right\|>1\right\} \cdots$

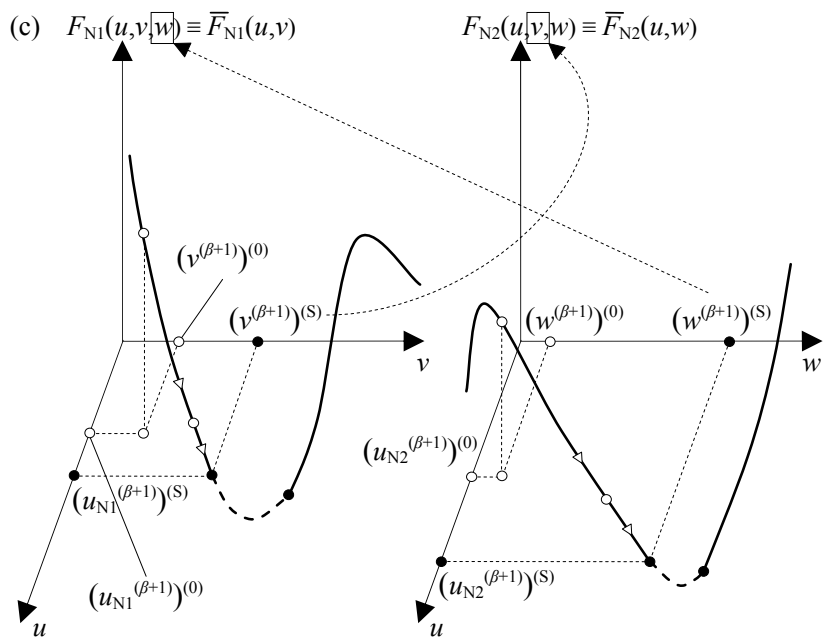

Figure 2. Numerical methods: (a) Newton-Raphson method. (b) Fixed-point iteration method. (c) Iteration $\beta+1$ of SHLF fixed-point iteration method. 
(a)

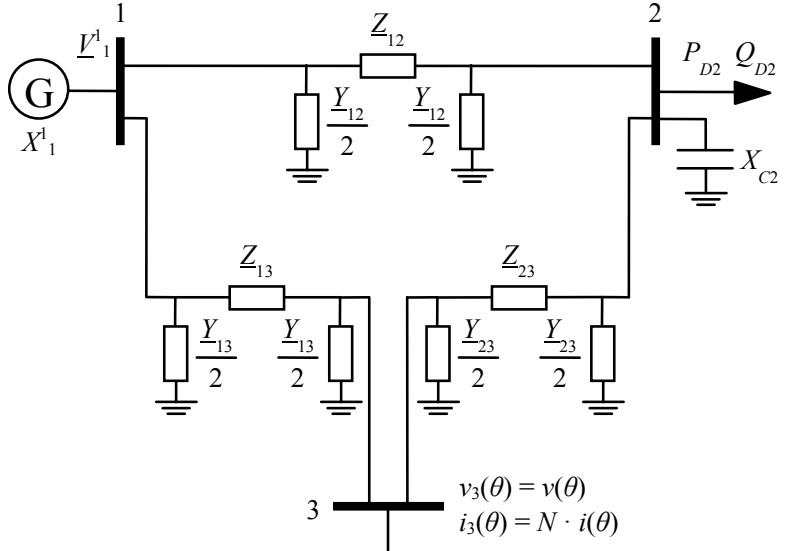

$N$ single-phase uncontrolled rectifiers

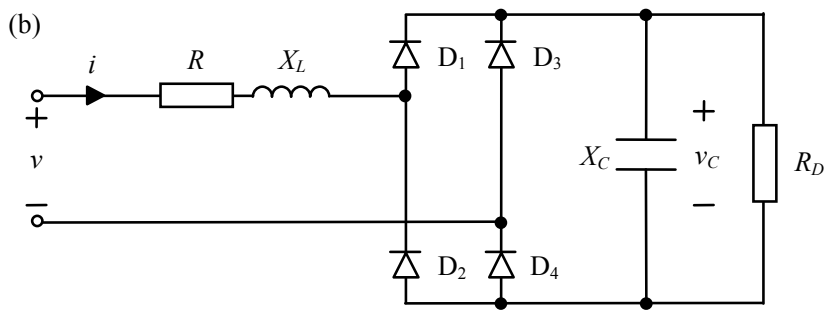

$v(\theta)=\sqrt{2} \cdot V^{1} \cos \left(\theta+\phi_{V^{1}}\right)+\sum_{h=3}^{5,7, \ldots} \sqrt{2} \cdot V^{h} \cos \left(h \theta+\phi_{V^{h}}\right), \quad \theta=\omega \cdot t$

(c)

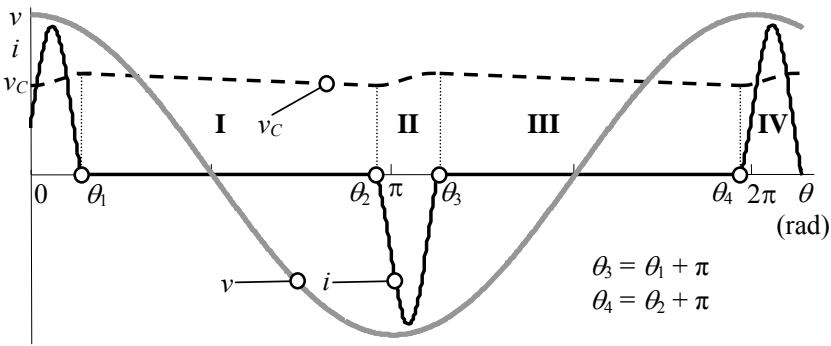

Figure 3. (a) Three-bus network. (b) Single-phase uncontrolled rectifier circuit. (c) Supply voltage $v$, ac current $i$ and dc voltage $v_{C}$ waveforms. 

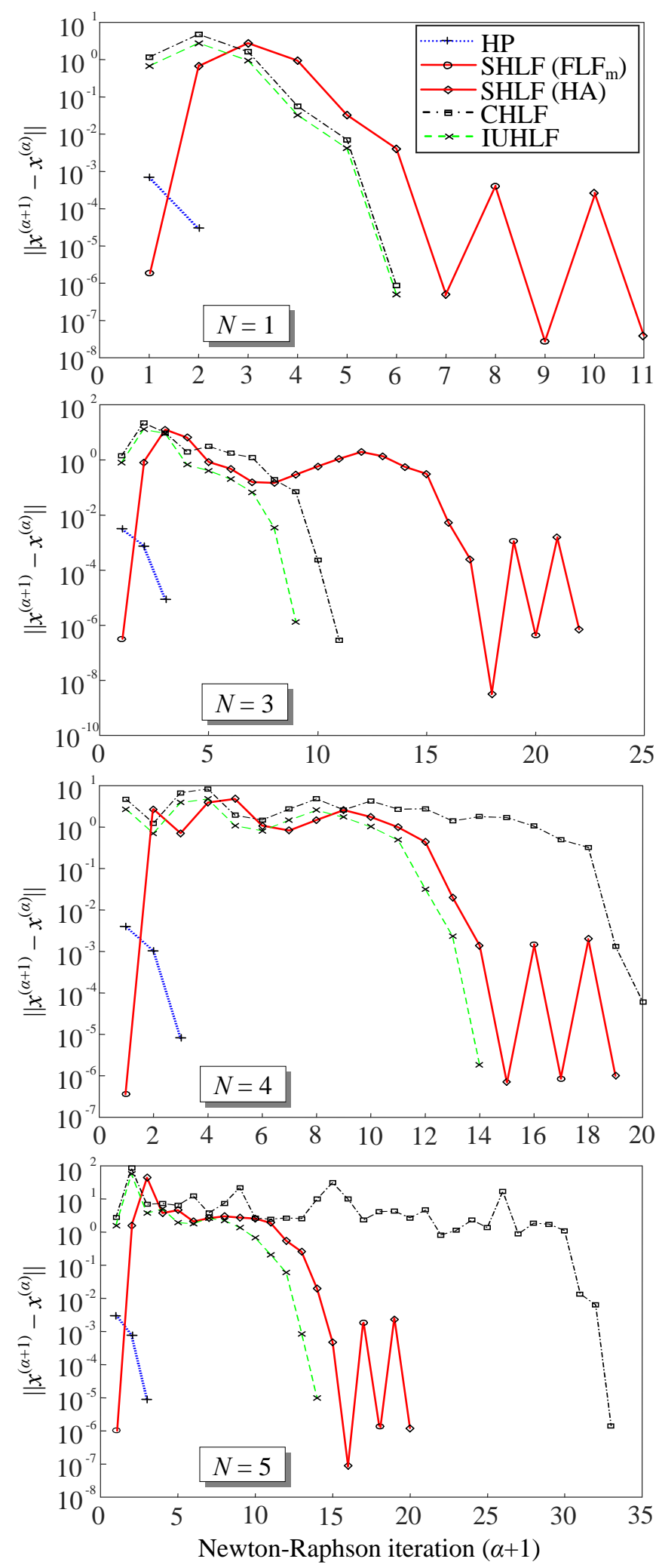


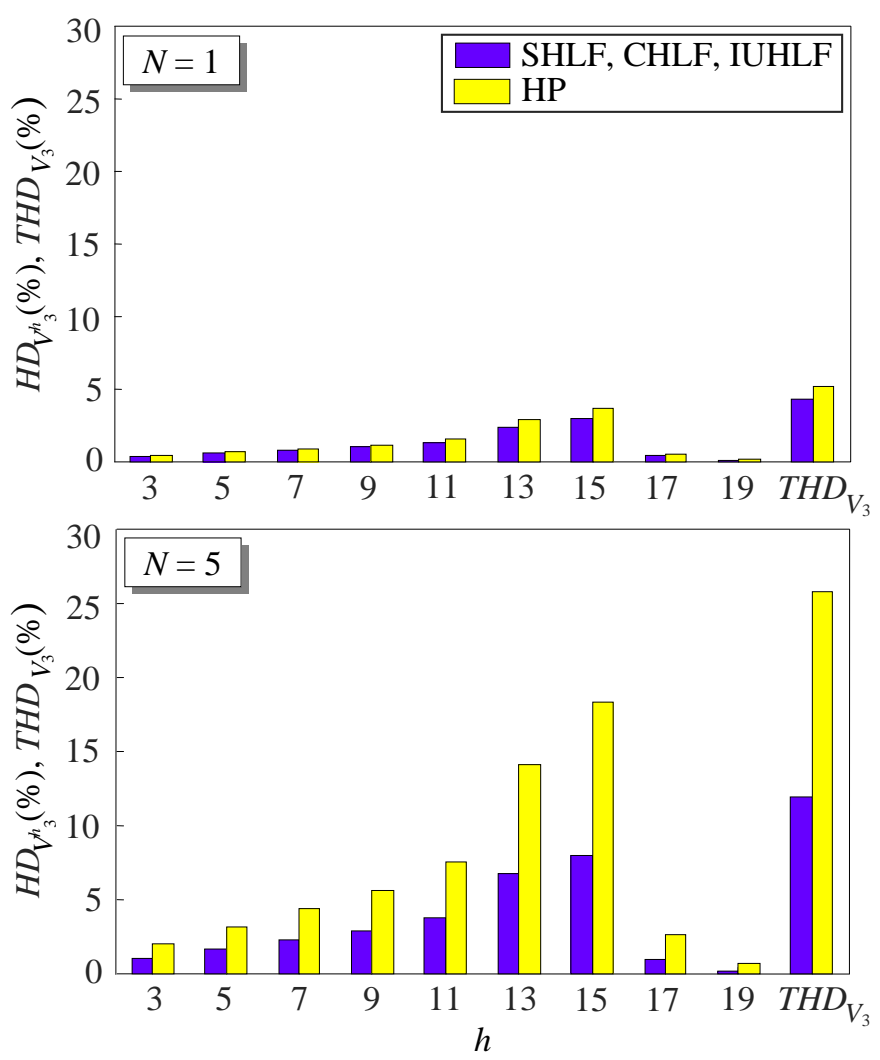


Tables

\begin{tabular}{|c|c|c|c|c|}
\hline Formulation & Stage & Bus & Data & Unknowns \\
\hline \multirow{2}{*}{ 主 } & 岳 & $\begin{array}{c}\text { Slack } \\
\text { PQ } \\
\text { NL }\end{array}$ & $\begin{array}{c}\underline{V}_{1}^{1} \\
P_{i}, Q_{i} \\
D_{i}^{m}\end{array}$ & $\begin{array}{c}-- \\
\underline{V}_{i}^{1} \\
\underline{\underline{V}}_{i}, \delta^{r}\end{array}$ \\
\hline & $z$ & $\begin{array}{c}\text { Slack } \\
\text { PQ } \\
\text { NL } \\
\end{array}$ & $\begin{array}{c}X_{1}^{1} \\
\underline{Y}_{i}^{h}=f_{{ }^{h}}^{h}\left(V^{1}{ }_{i}\right) \\
\underline{I}_{i}^{h}\end{array}$ & $\begin{array}{l}\underline{V}^{h}{ }_{1} \\
\underline{V}_{i}^{h} \\
\underline{V}_{i}^{h}\end{array}$ \\
\hline \multirow{3}{*}{ 崫 } & 王豆 & $\begin{array}{c}\text { Slack } \\
\text { PQ } \\
\text { NL }\end{array}$ & $\begin{array}{c}\underline{V}_{1}^{1} \\
P_{i}, Q_{i} \\
\underline{V}_{i, \mathrm{~N} 2}^{h}, D^{m}{ }_{i}\end{array}$ & $\begin{array}{c}--- \\
\underline{V}_{i, \mathrm{~N} 1}^{1} \\
\underline{V}_{i, \mathrm{~N} 1}^{1}, \delta^{r}{ }_{i, \mathrm{~N} 1} \\
\end{array}$ \\
\hline & 死胥 & $\begin{array}{c}\text { Slack } \\
\text { PQ } \\
\text { NL } \\
\end{array}$ & $\begin{array}{c}\underline{V}_{1}^{1}, X_{1}^{1} \\
\underline{Y}_{i}^{k}=f_{Y i}^{k}\left(V_{i, \mathrm{~N} 1}^{1}\right) \\
D_{i}^{m}{ }_{i}\end{array}$ & $\begin{array}{c}-- \\
-- \\
\underline{V}_{i, \mathrm{~N} 2}^{1}, \underline{V}_{i, \mathrm{~N} 2}^{h}, \delta_{i, \mathrm{~N} 2}^{r}\end{array}$ \\
\hline & z & $\begin{array}{c}\text { Slack } \\
\text { PQ } \\
\text { NL }\end{array}$ & $\begin{array}{c}X_{1}^{1}{ }_{1} \\
\underline{Y}_{i}^{h}=f_{Y i}^{h}\left(V_{i, \mathrm{~N} 1}^{1}\right) \\
\underline{I}_{i}^{h}\end{array}$ & $\begin{array}{l}\underline{V}^{h}{ }_{1} \\
\underline{V}^{h} \\
---\end{array}$ \\
\hline 鸢 & 岑 & $\begin{array}{c}\text { Slack } \\
\mathrm{PQ} \\
\mathrm{NL} \\
\end{array}$ & $\begin{array}{c}\underline{V}^{1}, X^{1}{ }_{1} \\
P_{i}, Q_{i} \\
D^{m}{ }_{i} \\
\end{array}$ & $\begin{array}{c}\underline{V}^{h}{ }_{1} \\
\underline{V}_{i}^{1}, \underline{V}_{i}^{h} \\
\underline{V}_{i}^{1}, \underline{V}_{i}^{h}, \delta_{i}^{r} \\
\end{array}$ \\
\hline 茪 & 焉 & $\begin{array}{c}\text { Slack } \\
\text { PQ } \\
\text { NL } \\
\end{array}$ & $\begin{array}{c}\underline{V}_{1}^{1} \\
P_{i}, Q_{i} \\
D^{m}{ }_{i} \\
\end{array}$ & $\begin{array}{c}--- \\
\underline{V}_{i}^{1} \\
\underline{V}_{i}^{1}, \underline{V}_{i}^{h}, \delta^{r}{ }_{i}\end{array}$ \\
\hline 窋 & $z$ & $\begin{array}{c}\text { Slack } \\
\text { PQ } \\
\text { NL } \\
\end{array}$ & $\begin{array}{c}X_{1}^{1} \\
\underline{Y}_{i}^{h}=f^{h}{ }_{{ }_{i}}\left(V^{1}{ }_{i}\right) \\
\underline{I}_{i}^{h}\end{array}$ & $\begin{array}{l}\underline{V}_{1}^{h} \\
\underline{V}_{i}^{h} \\
-- \\
\end{array}$ \\
\hline \begin{tabular}{ll}
\multicolumn{2}{l}{ Notes: } \\
- & Slack bus \\
- & NLL dat \\
- & Harmoni \\
& $k=1,3$,
\end{tabular} & $\begin{array}{l}m=1 t \\
\text { orders: }\end{array}$ & $\begin{aligned} & n_{\max } ; 1 \\
= & 3,5, .\end{aligned}$ & $\begin{array}{l}=2 \text { to } c \text {; NL bu } \\
\text { L state variable } \\
; \text { Fundamental } a\end{array}$ & $\begin{array}{l}: i=c+1 \text { to } n \\
=1 \text { to } r_{\max } \\
\text { harmonic orders: }\end{array}$ \\
\hline
\end{tabular}

Table 1. HLF formulations. 


\begin{tabular}{|c|c|c|c|c|}
\hline Element & \# & Type & Data & Values (p.u.) \\
\hline \multirow{3}{*}{ Bus } & 1 & Slack & $\frac{V^{1}}{X_{1}^{1}}{ }_{1}$ & $\begin{array}{c}1 \angle 0^{\circ} \\
4.6168 \mathrm{e}-3 \\
\end{array}$ \\
\hline & 2 & PQ & $\begin{aligned} P_{2} & =-P_{D 2} \\
Q_{2} & =-Q_{D 2}\end{aligned}$ & $\begin{array}{l}-0.8 \\
-0.6\end{array}$ \\
\hline & 3 & NL & $\begin{array}{c}R, X_{L} \\
X_{C}, R_{D} \\
\end{array}$ & $\begin{array}{c}0.0207,0.0130 \\
1.1579,11.0021 \\
\end{array}$ \\
\hline Shunt & $2-0$ & Capacitor & $X_{C 2}$ & 1.6667 \\
\hline \multirow{3}{*}{ Branch } & $1-2$ & Line & $\begin{array}{l}\underline{Z}_{12} \\
\underline{Y}_{12}\end{array}$ & $\begin{array}{c}5.1653 \mathrm{e}-4+j 5.1653 \mathrm{e}-3 \\
j 6.4533 \mathrm{e}-3 \\
\end{array}$ \\
\hline & $2-3$ & Line & $\begin{array}{l}\underline{Z}_{23} \\
\underline{Y}_{23}\end{array}$ & $\begin{array}{c}5.1653 \mathrm{e}-4+j 5.1653 \mathrm{e}-3 \\
j 6.4533 \mathrm{e}-3 \\
\end{array}$ \\
\hline & $1-3$ & Line & $\begin{array}{l}\underline{Z}_{13} \\
\underline{Y}_{13} \\
\end{array}$ & $\begin{array}{c}5.1653 \mathrm{e}-4+j 5.1653 \mathrm{e}-3 \\
j 6.4533 \mathrm{e}-3 \\
\end{array}$ \\
\hline \begin{tabular}{cc}
\multicolumn{2}{l}{ Notes: } \\
- & Base \\
- & Funn
\end{tabular} & lues & $\begin{array}{l}=2500 \\
\text { requency }\end{array}$ & $\begin{array}{l}V_{\mathrm{B}}=220 \mathrm{~V} \\
\text { e supply sys }\end{array}$ & $: f=50 \mathrm{~Hz}$ \\
\hline
\end{tabular}

Table 2. Network data. 